\title{
AVALIAÇÃO NUTRICIONAL DE LACTENTES HOSPITALIZADOS
}

\section{NUTRITIONAL EVALUATION OF HOSPITALIZED SUCKLING CHILDREM}

\author{
João Guilherme Bezerra Alves* \\ José Eulálio Cabral Filho ** \\ José Natal Figueiroa***
}

ALVES, JGB et al. Avaliação nutricional de lactentes hospitalizados. Rev Esc Enf USP, v.35, n, 1, p. 60-4, mar. 2001.

\begin{abstract}
RESUMO
Investigou-se a evolução ponderal, conforme o valor das quotas calóricas de dietas prescritas (QCP) e aceitas (QCA) de lactentes internados por diarréia ou broncopnemonia no Instituto Materno Infantil de Pernambuco, Brasil. A proporção de crianças que ganharam ou não ganharam peso foi avaliada em função das QCP E QCA que atingiram as quotas calóricas ideais (QCI) durante o internamento, verificando-se que 46\% das crianças perderam ou mantiveram o peso nos dois primeiros dias. Houve maior freqüencia de ganho de peso associado às QCP e QCA que atingiram as QCI, bem como entre as crianças amamentadas. Concluiu-se existir deficiente evolução ponderal relacionada às baixas quotas calóricas oferecidas ou aceitas durante a hospitalização.
\end{abstract}

PALAVRAS-CHAVE: Desnutrição. Ingestão calórica. Criança. Hospitalização.

\begin{abstract}
This work investigated the body weight evolution according to prescribed or accepted dietetic caloric value, in children with diarrhea or bronchopneumonia, admitted at Instituto Materno Infantil de Pernambuco, Brazil. The proportions of children who increased or did not increase body weight were evaluated as a function of the prescribed caloric quota (PCQ) and the accepted caloric quota (ACQ) that attained the ideal caloric quota (ICQ) during hospitalization. 46\% children lost or maintained the weight during the two first days. A higher frequency of increased weight was observed when PCQ or ACQ attained the ICQ, as well as in breast feeding children. Therefore, this study confirmed the relationship between low caloric values offered or accepted with a deficient body weight evolution.
\end{abstract}

KEYWORDS: Malnutrition. Caloric intake. Children. Hospitalization.

\section{INTRODUÇÃO}

A nutrição é um dos principais aspectos a levarse em consideração na recuperação de enfermos, sendo a evolução ponderal um critério relevante na sua avaliação. Entretanto os efeitos da hospitalização sobre o estado nutricional de 'pacientes têm sido pouco estudados, desconhecendo-se assim a sua real importância (PANTIN, 1982; LARSSON, 1993; DICKERSON, 1995). Dentre os primeiros autores a tratar deste tema estão PINCHOFSKY e KAMINSKI (1985) que relataram efeitos prejudiciais da hospitalização sobre o estado nutricional. Só há poucos anos, editoriais e cartas ao editor têm chamado a atenção para a desnutrição energético-protéica (DEP) de aquisição intrahospitalar (HEANERY, 1993; AVENELL,1994; GARROW, 1994; ROYCE; TAYLOR, 1994). Por outro lado a maior parte dos estudos tem sido realizada em adultos e em países ricos, e tem indicado um papel adverso da hospitalização no ganho de peso e na nutrição dos enfermos (ARMELINI et al, 1982; SHAW, 1993; MAINOUS; DEITCH, 1994; MILLER; MILLER, 1994). E pequeno o número de trabalhos que indicam o contrário (REYNA; OSCAR, 1986; COATS et al 1993).

O problema é ainda menos estudado nas regiões pobres, onde as taxas de desnutrição energéticoprotéica são elevadas. Nessas regiões a DEP contribui

* Professor, Instituto Materno Infantil de Pernambuco, MD. E-mail: joaoguilherme@imip.org.br

** Professo, Instituto Materno Infantil de Pernambuco, DSc

*** Professor, Instituto Materno Infantil de Pernambuco, MSc, 
para uma contínua demanda dos serviços de saúde, especialmente daqueles destinados a atendimento infantil. No Nordeste do Brasil, as formas graves de DEP chegam a atingir $24 \%$ das crianças hospitalizadas, menores de 5 anos de idade ( ROCHA et al, 1981; BATISTA, FILHO; COSTA 1988). Essa elevada freqüência evidencia, de forma contundente, o papel relevante do hospital como centro de demanda dos casos de DEP grave. O não reconhecimento desses casos, paralelamente à falta de suporte nutricional adequado, tende a agravar sensivelmente o prognóstico dos mesmos.

A possibilidade da deficiência nutricional ser gerada dentro do hospital é um fato grave e da maior importância, principalmente pelas implicações sócioeconômicas que tem sobre o campo da saúde, tais como a vinculação com elevadas taxas de morbimortalidade, a maior permanência hospitalar e a elevação do custo do leito (ROBINSON et al, 1987; LENNARD-JONES, 1992). Teoricamente as crianças mais jovens, dada a sua maior velocidade de crescimento e necessidades metabólicas, são mais susceptiveis à desnutrição durante o internamento. Isto é tanto mais provável quando são vítimas de processos infecciosos, os quais aumentam grandemente suas necessidades energéticas e reduzem a aceitação alimentar tendo como provável conseqüência uma redução ponderal.

Mesmo com a prescrição de quotas calóricas adequadas $(\mathrm{QCA})$, existe o risco das crianças não aceitarem a dieta pois além da agressão imposta ao hospedeiro pelo agente infeccioso, outros fatores podem contribuir para que a dieta prescrita não seja totalmente aceita: o horário em que a alimentação é servida, o ambiente em que são oferecidas as refeições, sabor e a forma de apresentação, entre outros.

Dessa forma, foi objetivo do presente estudo verificar a evolução ponderal de lactentes hospitalizados com diarréia ou broncopneumonia, analisando o valor calórico das dietas prescritas e aceitas que atingiram as quotas calóricas ideais.

\section{MATERIAL E MÉTODOS}

O estudo, realizado no período 1997-1998, envolveu 300 lactentes (168 meninas e 132 meninos) com idade entre 1 e 11 meses, (mediana $=3$ meses), internados no Instituto Materno Infantil de Pernambuco (IMIP), entidade credenciada pelo Ministério da Educação como hospital de ensino, e que atende a $7 \%$ da população materno-infantil do estado de Pernambuco, Nordeste do Brasil. A maioria $(50,7 \%)$ era proveniente de famílias com renda "per capita" inferior a US\$30,00 (trinta dólares americanos). Todos os casos foram internados com diarréia aguda (165 crianças) ou broncopneumonia
(135 crianças) e tinham história da doença de até 7 dias de evolução. Foram incluídas apenas crianças com alimentação exclusivamente láctea durante a hospitalização, e excluídas aquelas portadoras de malformações congênitas, doenças metabólicas, edema ou forma grave de desnutrição protéico energética, insuficiência respiratória, espasticidade brônquíca ou sinais de choque. Os dados foram obtidos através de entrevista com as acompanhantes, prescrições médicas anotadas nos prontuários e medidas antropométricas verificadas pelos autores. Os dados antropométricos foram obtidos com a criança clinicamente hidratada, despida e sem a bexiga palpável. Considerou-se como medida do peso a média aritmética de três aferições realizadas em torno de 1 hora após a alimentação. As duas primeiras foram coletadas independentemente, por dois médicos assistentes das enfermarias, usando uma balança tipo "pesa-bebê" (marca Filizola, modelo 30) e a terceira foi realizada pelo autor principal, com balança eletrônica (marca SOEHNLE-WAAGHEN GMBH, Murrahardt, Germany modelo 5011).

O acompanhamento foi realizado a partir do momento da admissão hospitalar até a ocasião da alta ou óbito, sendo a permanência hospitalar de 2 a 12 dias, com média de 4,9 $\pm 1,3$ dias. Diariamente eram registrados o peso corporal, bem como a dieta prescrita e a aceita por cada criança. A informação sobre a dieta prescrita era obtida através do prontuário médico e a informação sobre a aceitação, através da genitora.

Para cada criança foi calculada a quota calórica ideal (QCI), de acordo com a idade, segundo recomendação da "National Academy of Sciences" (Food and Nutritional Board, 1980). As quotas calóricas prescritas (QCP) e as aceitas (QCA) foram calculadas a partir do volume da fórmula láctea prescrita, considerando-se o valor de 670 Kcal para cada litro.

As crianças com idade inferior a 5 meses e que não estavam recebendo amamentação exclusiva, foram relactadas conforme orientação da equipe do Centro de Incentivo ao Aleitamento Materno do IMIP. Foram considerados relactados aqueles que passaram a receber exclusivamente o leite materno, sem nenhum tipo de complemento.

Neste estudo foram computados os dados dos 5 primeiros dias de internamento, desde que este é o tempo médio de permanência de crianças com broncopneumonia e diarréia no IMIP.

A associação de variáveis foi avaliada pelo teste Qui-quadrado, admitindo-se o nivel de significância de a $£ £ 0,05$. Na determinação do risco relativo foi calculada a "odds ratio"(OR) com um intervalo de confiança de 95\% (1C95). As análises estatísticas foram executadas através dos "softwares" Epi-Info versão 6.0 e Statistic. 
Após as devidas informações para esclarecimentos, a permissão para inclusão de cada criança na pesquisa foi dada pelos seus responsáveis (pais ou acompanhantes), conforme solicitação de rotina para todos os pacientes internados no IMIP o qual autorizou a realização da mesma .

\section{RESULTADOS}

Durante os dois primeiros dias de hospitalização, 162 (54\%) crianças ganharam peso, enquanto $138(46 \%)$ apresentaram peso igual ou inferior ao da admissão.
Exceto no $4^{\circ}$ dia de internamento, as proporções de crianças cujas QCP atingiram as quotas ideais foram significantemente maiores no grupo com ganho de peso do que no grupo sem ganho de peso (Tabela 1). As proporções de crianças cujas QCA atingiram as ideais também foram significantemente maiores no grupo com ganho de peso do que no grupo sem ganho de peso, exceto no $5^{2}$ dia de internamento (Tabela 2).

Entre os dias 3 e 5 ocorreram 20 (14,5\%) óbitos entre os lactentes que perderam ou mantiveram o peso e $10(6,2 \%)$ entre os que aumentaram de peso $(p=0.027)$. A proporção de altas nos dois grupos foi , praticamente a mesma: 86 (53.1\%) no grupo com ganho de peso e 73 (52.8\%) no outro grupo.

Tabela 1- Evolução ponderal dos lactentes cujas quotas calóricas prescritas atingiram as ideais.

IMIP, Recife, 1997-1998

\begin{tabular}{|c|c|c|c|c|c|c|c|c|}
\hline $\begin{array}{c}\text { Dia de } \\
\text { internamento }\end{array}$ & $\begin{array}{c}\text { Lactentes } \\
\text { sem ganho de }\end{array}$ & $\%$ & $\begin{array}{c}\text { Lactentes com } \\
\text { ganho de } \\
\text { peso }\end{array}$ & $\%$ & $\mathrm{X}^{2}$ & $a$ & OR & IC 95 \\
\hline 1 & $43 / 138^{*}$ & 31 & $73 / 162$ & 45 & 5,50 & 0,019 & 0,55 & $(0,33-0,91)$ \\
\hline 2 & $58 / 138$ & 42 & $102 / 162$ & 63 & 12,29 & 0,000 & 0,43 & $(0,26-0,70)$ \\
\hline $3^{* *}$ & $65 / 128$ & 51 & $114 / 158$ & 72 & 12,89 & 0,000 & 0,40 & $(0,24-0,67)$ \\
\hline 4 & $46 / 75$ & 61 & $74 / 105$ & 70 & 1,26 & 0,261 & 0,66 & $(0,34-1,30)$ \\
\hline 5 & $26 / 45$ & 58 & $48 / 66$ & 73 & 5,22 & 0,022 & 0,37 & $(0,16-0,88)$ \\
\hline
\end{tabular}

Tabela 2 - Evolução ponderal dos lactentes cujas quotas calóricas aceitas atingiram as ideais. IMIP , Recife, 1997 - 1998

\begin{tabular}{|c|c|c|c|c|c|c|c|c|}
\hline $\begin{array}{c}\text { Dia de } \\
\text { internamento }\end{array}$ & $\begin{array}{c}\text { Lactentes } \\
\text { sem ganho de } \\
\text { peso }\end{array}$ & $\%$ & $\begin{array}{c}\text { Lactentes com } \\
\text { ganho de } \\
\text { peso }\end{array}$ & $\%$ & $\mathrm{X} 2$ & $\mathrm{a}$ & OR & IC 95 \\
\hline 1 & $11 / 138^{*}$ & 08 & $44 / 162$ & 27 & 17,07 & 0,000 & 0,23 & $(0,11-0,49)$ \\
\hline 2 & $30 / 138$ & 22 & $79 / 162$ & 49 & 22,4 & 0,000 & 0,29 & $(0,17-0,50)$ \\
\hline $3 * *$ & $37 / 128$ & 29 & $97 / 158$ & 61 & 28,7 & 0,000 & 0,26 & $(0,15-0,43)$ \\
\hline 4 & $24 / 75$ & 32 & $69 / 105$ & 66 & 18,6 & 0,000 & 0,25 & $(0,12-1,48)$ \\
\hline 5 & $14 / 45$ & 31 & $16 / 66$ & 24 & 0,34 & 0,560 & 0,23 & $(0,10-0,49)$ \\
\hline
\end{tabular}

Numerador: número de lactentes com QCA = QCI; denominador: total de lactentes no grupo "

Redução do número de lactentes do $3^{\circ}$ ao $5^{\circ}$ dia, por alta ou por óbito

Comparando-se as freqüências de ganho ou perda de peso entre os lactentes que recebiam ou não o leite materno (Tabela 3), observou-se freqüência significativamente maior de crianças sem ganho de peso entre estes últimos $(\mathrm{a}=0,000 ; \mathrm{OR}=0,17 ; \mathrm{IC}$ $95=0,07-0,42)$.

Tabela 3 - Evolução ponderal dos lactentes segundo presença ou não de aleitamento materno. IMIP, Recife, 1997 - 1998.

\begin{tabular}{ccccccccccc}
\hline $\begin{array}{c}\text { Aleitamento } \\
\text { materno }\end{array}$ & $\begin{array}{c}\text { Lactentes } \\
\text { sem ganho } \\
\text { de } \\
\text { peso }\end{array}$ & $\%$ & $\begin{array}{c}\text { Lactentes } \\
\text { com ganho } \\
\text { de } \\
\text { peso }\end{array}$ & $\%$ & Total & $X^{2}$ & a & OR & IC 95 \\
\hline SIM & 7 & 7,6 & 42 & 32,6 & 49 & 17,95 & 0,000 & 0,17 & $(0,07-0,42)$ \\
NÃO & 85 & 92,4 & 87 & 67,4 & 172 & \\
\hline
\end{tabular}




\section{DISCUSSÃO}

Os achados deste estudo revelaram que a afirmação de que a perda de peso é inevitável durante a hospitalização não é verdadeira, pois mostraram que o ganho de peso foi maior nos pacientes cujas quotas calóricas aceitas (ou prescritas) atingiram os valores ideais, como esperado. PENNINGTON (1997), estudando um grupo de pacientes internados, também verificou que quando se assegurava uma cobertura minima da quota calórica necessária ou requerida, a perda de peso, era interrompida.

No presente estudo, 46\% dos lactentes perderam ou mantiveram o peso corporal no inicio da hospitalização, não tendo assegurada a manutenção do crescimento pleno. Segundo Bernstein et al (1993), 30 a $50 \%$ dos pacientes hospitalizados têm possibilidade de desenvolver denutrição intra hospitalar independentemente da sua condição clínica. MCWHIRTER e PENNINGTON (1994) constataram que dois terços dos 500 pacientes adultos por eles estudados apresentaram redução de peso durante o internamento. No entanto, em lactentes, DUFFAUTORO et al (1986) observaram deterioração do estado de nutrição em apenas 3 a 5\% de lactentes hospitalizados por patologia respiratória ou digestiva, no Chile. No Brasil, por outro lado, ROCHA et al (1997) observaram que $27,8 \%$ dos lactentes internados no Instituto de Puericultura e Pediatria Martagão Gesteira (RJ) perderam peso durante a hospitalização. Não se pode excluir a possibilidade de que a perda ou apenas a manutenção do peso inicial se deva, pelo menos em parte, à adaptação ao "stress"da hospitalização. Mas a associação, durante no minimo 4 dias, do ganho em peso da maioria das crianças cujas quotas calóricas prescritas e as aceitas atingiram as quotas calóricas ideais, sugere que estas ofertas alimentares são fatores fundamentais na recuperação nutricional dos pacientes.

Mais importante que a inadequação das quotas calóricas em pacientes hospitalizados é a verificação de sua aceitação, pois o quadro de anorexia que geralmente acompanha as infecções leva à não aceitação dos alimentos oferecidos, constituindo um dos principais fatores que determinam o balanço energético negativo. No presente estudo, apenas metade dos lactentes ingeriu a quantidade de calorias prescritas no primeiro dia de hospitalização. Essa aceitação foi significativamente diferente entre os pacientes que se subnutriram e os que não se subnutriram durante $o$ internamento. MCWHIRTER e PENNINGTON (1994) também verificaram, em um hospital universitário, que menos da metade dos pacientes recebia os nutrientes necessários, seja por via oral ou parenteral, devido a erros nas prescrições ou na sua execução; porem quando o suporte nutricional era adequado, havia melhoria do estado nutricional. Estudando idosos, KLIPSTEIN-
GROBUSCH et al (1995) apontaram o balanço energético negativo como o principal fator de risco para a subnutrição hospitalar. INCALZI et al (1996), por sua vez, detectaram que menos de $40 \%$ dos idosos ingeriam a quota calórica prescrita, e que a depleção nutricional observada durante o internamento associava-se diretamente com essa deficiência. Para esses autores, a modificação na qualidade dos alimentos, no modo de ofertá-los, e no estabelecimento de horários adequados para cada paciente, poderiam melhorar o comportamento nutricional durante o internamento.

Um dos resultados mais importantes do presente estudo foi a constatação de que o aumento de peso é significantemente maior entre as crianças em aleitamento materno. Uma explicação para tal fato, afora a ação antiinfecciosa do leite materno, especialmente nas infecções do trato digestivo e sistema respiratório (EBRAHIM, 1995; BEAUDRY et al 1995) poderia residir na melhor aceitação alimentar destas crianças. Existem evidências de que quando anoréticos, os lactentes recusam alimentos sólidos, porém continuam a mamar (DICKIN; BROWN, 1990). Hoyle (1980) verificou que o quadro de anorexia, induzido pela doença diarréica, é melhor controlado com o leite materno.

A elevada freqüência de desnutrição encontrada entre as crianças estudadas era esperada pelas condições inerentes aos quadros infecciosos aqui considerados. Entretanto, os resultados indicam que uma manipulação dietética mais adequada, não só quanto à prescrição, como à tentativa de aumentar a aceitação alimentar, pode contribuir para diminuir esse grave problema (SUAREZ, 1988; COMMITEE ON NUTRITION OF THE BRITISH PAEDIATRIC ASSOCIATION, 1994).

Pela importância do fator nutricional na manutenção e recuperação da saúde da criança, especialmente frente às condições patológicas mais freqüentes em nosso meio, isto é, diarréia e broncopneumonia, há necessidade de maior atenção à prescrição dietética. Sugere-se que as crianças que não recebem oferta alimentar livre de acordo com a aceitação, tenham o cálculo de sua quota calórica necessária calculada diariamente pelo médico acompanhante, tal como é feita para as quotas hidroeletroliticas. Ademais os acompanhantes devem receber orientação acerca da importância de se oferecer uma elevada quota calórica à criança nessa fase, com atenção especial de auxiliares de enfermagem. Essas medidas contribuirão para uma oferta alimentar mais cuidadosa que certamente assegurará uma melhor aceitação da dieta.

Dada a natureza e a importância do problema, é evidente a necessidade da implementação de novas investigações, com a utilização de outros métodos de pesquisa que permitam a realização de análises mais completas sobre as suas causas, de forma a contribuir efetivamente para a sua prevenção. 


\section{REFERÊNCIAS BIBLIOGRÁFICAS}

ARMELINI, PA et al. Evaluación prospectiva del estado nutricional del nino hospitalizado. Arch ArgentPediatr, v.80, n.2, p.213-7,1982.

AVENELL, A. Starvation in hospital. Ethically indefensible and expensive [letter]. BMJ, v.308, n.6940, p.1369, 1994.

BATISTA FILHO, M.; COSTA, MJC. Desnutrição enérgeticoprotéica: experiência do Hospital Universitário de João Pessoa, Pb. I- Prevalência da desnutrição. Rev IMIP, v.2, n.2, p 90-3, 1988.

BEAUDRY, M.; DUFOUR, R.; MARCOUX, S. Relation between infant feeding and infections during the first six months of life. J Pediatr, v.162, n.2, p.191-7, 1995.

BERNSTEIN, LH et al. Financial implications of malnutrition. Clin Lab Med, v.13, n.2, p.491-507, 1993.

COATS, KG et al. Hospital associated malnutrition: a reevaluation 12 years later. J Am Diet Assoc, v.93, n.1, p.2'7-33, 1993

COMMITTEE ON NUTRITION OF THE BRITISH PAEDIATRIC ASSOCIATION. Is breast feeding beneficial in the UK? Statement of the standing. Arch Dis Child, v.71, n.4, p.376-80, 1994.

DICKERSON, J. The problem of hospital-induced malnutrition. Nurs Times, v.91, n.4, p.44-5, 1995.

DICKIN, KL.; BROWN, KH. Effect of diarrhoea on dietary intake by infants and young children in rural villages of Kwara State, Nigeria. Eur J Clin Nutr, v.44, p.307-18, 1990.

DUFFAU-TORO, G.; LOYOLA, JF; SEPÚlVEDA, PO. Estado nutricional del lactente hospitalizado por patologia respiratoria y digestiva. Estudio prospectibo. Bol Med Hosp Inf México, v.43, n.1, p.11-23, 1986.

EBRAHIM, GJ. Breast and immunology. $J$ Trop Pediatr, v.41, p.2-4, 1995

FOOD AND NUTRITIONAL BOARD NATIONAL ACADEMY OF SCIENCIES. National Research Council Recommended Daily Dietary Allowances, Revised, 1980.

GARROW, J. Starvation in hospital. Nutrition is given too little attention by doctors, nurses, and managers. BMJ, v.308, n.6936, p.934-5, 1994.

HEANERY, RP. More thoughts on hospital malnutrition: the skeleton in the closet [letter]. J Am Diet Assoc, v.93, n.4, p.400, 1993.

HOYLE, B. Breast-feeding and food intake among children with acute diarrheal disease. AmJ Clin Nutr,v.33, n.11, p.2365-71, 1980.

INCALZI, RA et al. Energy intake and in-hospital starvation. A clinically relevant relationship. Arch Int Med, v.156, n.26, p.425-9, 1996.
KLIPSTEIN-GROBUSCH, K. et al. Energy intake and expediture in elderly patients admitted to hospital with acute illness. BMJ, v.73, n.2, p.323-4, 1995.

LARSSON, J. Clinical problem of hospital malnutrition [editorial]. Nutr, v.9, n.3, p.274-5, 1993.

LENNARD-JONES, JE. A prospective approach to nutrition as treatment. London: King's Fund,1992.

MAINOUS, MR.; DEITCH, EA. Nutrition and infection. Surg Clin N Am, v.74, n.3, p.659-76, 1994.

McWHIRTER, JP.; PENNINGTON, CR. Incidence and recognition of malnutrition in hospital. BMJ, v.308, n,9, p.945-8, 1994.

MILLER, S.; MILLER, JM. Starvation in Hospital. Malnutrition goes unnoticed before surgery [letter]. BMJ, v.308, n.6940, p.1369, 1994.

PANTIN, EL. Desnutricion hospitalaria: un nuevo problema? Isabelica Med, v.7, n.1/2, p.97-106, 1982.

PENNINGTON, CR. Patients go hungry in British hospitals. BMJ, v.314, n.8, p.752, 1997.

PINCHOFSKY GD.; KAMINSKI, MV. Increasing malnutrition during hospitalization: documentation by a nutritional screening program. J Am Coll Nutr, v.4, p.471-9, 1985.

REYNA, P.; OSCAR, G. Efecto de la hospialización sobre el estado nutritional. Diagnóstico (Peru), v.18, n.1, p.25-9, 1986.

ROBINSON, G.; GOLDSTEIN, M.; LEVINE, GM. Impact of nutritional status on DRG lenght of stay. $J$ Parenter Enteral Nutr, v.11, p.49-51, 1987.

ROCHA, HF.; BRUNO, LE.; SANTOS, S. Avaliação sobre a variação de peso em lactentes internados no IPPMG-UFRG no período de janeiro de 1996 a maio de 1997. Arq Bras Pediatr, v.4, n.1, p.61, 1997.

ROCHA, JA.; TOSCANO, G.; OLIVEIRA I. Desnutrição e Hospital. I-Prevalência de desnutrição protéico-calórica em crianças hospitalizadas. JPediatr, v.51, n.2, p.106-8, 1981.

ROYCE, C.; TAYLOR, M. Starvation in Hospital. Identifying malnutrition benefits everybody [letter]. BMJ, v.308, n.6940, p.1370, 1994.

SHAW, STA et al. Effect of nutrition status and other factors on lenght of hospital stay after major gastrointestinal surgery. Nutr, v.9, n.3, p.140-5, 1993.

SUÁREZ, R et al. Malnutricion hospitalaria: Causas $y$ consequencias: propuesta para organizar um servicio de apoyo nutricional. CM Publ Med, v.1, n.1, p.12-7, 1988.

Artigo recebido em 04111199

Artigo aprovado em 15/05/01 